\title{
Análisis y perspectivas de las coberturas de acueducto y alcantarillado en el Departamento del Meta
}

\section{Analysis and perspectives regarding water supply and sewerage coverage in the Meta Department}

\section{Análise e perspectivas da cobertura de água e esgoto no Departamento del Meta.}

Hernando Castro-Garzón ${ }^{1}$, Miguel A. Rubio-Cruz ${ }^{2}$,Juan P. Rodríguez-Miranda ${ }^{3}$

\author{
Administrador de Empresas. Universidad de Los Llanos \\ Ingeniero Agrónomo. Universidad de Los Llanos. \\ Profesor Asociado. Facultad del Medio Ambiente y Recursos Naturales. Universidad Distrital Francisco José de Caldas. Bogotá, \\ D.C. \\ Email: jprodriguezm@udistrital.edu.co
}

\section{Resumen}

Se recolectó y analizó la información proveniente de los 29 municipios del Departamento del Meta, Colombia, los cuales fueron clasificados según la categoría y la cantidad de habitantes en función del porcentaje de cobertura de acueducto y alcantarillado, del índice de riesgo de la calidad del agua para consumo humano y de la instalación de plantas de tratamiento de aguas residuales. Los resultados señalan una cobertura de acueducto de $21.2 \%$, y de alcantarillado de $20.8 \%$. El 43\% de los municipios presentan una calidad del agua para el consumo humano con nivel de riesgo Inviable - Alto; $21 \%$ con riesgo bajo, y $18 \%$ sin riesgo. Además, el $68 \%$ de los municipios no cuentan con una planta de tratamiento de aguas residuales y solo el $32 \%$ realizan tratamiento de las mismas.

Palabras Clave: Coberturas, acueducto, alcantarillado, calidad del agua.

\begin{abstract}
This article collected Information from 29 municipalities in the department of Meta, Colombia, ranked were collected according to the category and the number of people, depending on the percentage coverage of water supply and sewerage, the risk index of water quality for human consumption and installation plant wastewater. Results show coverage aqueduct $21.2 \%$ and $20.8 \%$ sewerage. $43 \%$ of the municipalities have a water quality for human consumption of unworkable risk level - High, low risk $21 \%$ and $18 \%$ risk. In addition, $68 \%$ of municipalities do not have a treatment plant wastewater and only $32 \%$ made the same treatment.
\end{abstract}

Key words: Coverage, water supply, sewerage, water quality. 


\section{Resumo}

Foram coletadas e analisadas informações de 29 municípios, no departamento de Meta, na Colômbia, as quais foram classificadas segundo a categoria e a quantidade de pessoas em função da porcentagem de cobertura de abastecimento de água e esgoto, o índice de risco da qualidade da água para consumo humano e instalação tratamento de aguas residuais. Os resultados mostram uma cobertura de abastecimento de agua de $21,2 \%$ e $20,8 \%$ de esgoto. $43 \%$ dos municípios têm qualidade da água para consumo humano, de nível de risco inviável Alto, baixo risco de $21 \%$ e risco de $18 \%$. Além disso, $68 \%$ dos municípios não possuem uma estação de tratamento de águas residuais e só $32 \%$ fizeram o mesmo tratamento.

Palavras-chave: coberturas, aqueduto, esgoto, qualidade da água.

\section{Introducción}

Unas de las presiones importantes del crecimiento económico y de la explosión demográfica es, entre otras, la demanda por el agua en cantidad y calidad suficiente para abastecer las necesidades tanto del sector doméstico, el comercial, el industrial y hasta el agrícola. Sin embargo, en algunos casos, la disponibilidad es variable según la ubicación en el espacio o territorio (Nogueira, 2015; Roelich, 2015). En el caso de Colombia, en algunas regiones se presenta escasez, es decir, poca cantidad $y$, en otras, aunque exista una cantidad adecuada, la calidad del agua no es la recomendada (Rodríguez, 2010).

En Latinoamérica se estima que la dotación de agua promedio es de 209 I/hab. día; la población servida promedio con agua potable es del $85 \%$; la cobertura de alcantarillado de $77 \%$, y menos del $20 \%$ de las aguas residuales reciben tratamiento (CEPIS, 2000).

Además, los servicios públicos de agua en los países en desarrollo, se enfrentan a enormes desafíos en el cumplimiento de las necesidades de agua, por efecto de su crecimiento urbano (Nogueira, 2015). Estos desafíos son el resultado de prácticas inadecuadas en la gestión de servicios públicos domiciliarios, incluyendo una falta de cultura comercial y de organización
(Mugabi, 2007); sin dejar de mencionar la falta de voluntad política, la poca articulación de los planes de desarrollo municipales y la escasa visión de planificación y ordenamiento territorial, con respecto al saneamiento ambiental de los municipios.

Lo anterior tiene consecuencias para los países en vía de desarrollo, en donde el $10 \%$ de la carga total de las enfermedades en la población se atribuye a la falta de agua, saneamiento e higiene. Aquello favorece que el $60 \%$ de estas enfermedades sean de carácter hídrico (Norman, 2010; Fry, 2012).

Latinoamérica posee una población de 596 millones de personas, mientras en Colombia, se aproxima a 47 millones (OPS, 2012). El diagnóstico del sector de agua potable, presenta cifras importantes de coberturas de abastecimiento de agua de $91 \%$ en ciudades como Bogotá, Cali y Medellín; mientras en Barranquilla la cobertura supera el $95 \%$ y el saneamiento con cobertura del 85\% (Sánchez, 2013). Sin embargo, en los indicadores básicos de la Organización Panamericana de la Salud, nuestro país cuenta con un $92 \%$ de la población total con acceso a fuentes de agua potable, $99 \%$ en la zona urbana y $72 \%$ en la zona rural; y $77 \%$ de la población total con acceso a instalaciones de saneamiento, $82 \%$ en la zona urbana y $63 \%$ en la zona rural (OPS, 2012). 
Otros indicadores relevantes del sector, establecen que la capacidad per cápita de agua potable es de 0.004 I/habitante; la evolución de agua potable apta es del $35.6 \%$, y no apta del $64.4 \%$; el índice promedio de agua no contabilizada es de $42 \%$. La continuidad del servicio de acueducto es del $91.6 \%$, equivalente a 22 horas de suministro continuo por día (Marín, 2011). En un estudio sobre la situación de salud en Las Américas, se señala que la población colombiana tiene acceso a fuentes mejoradas de agua potable de $92 \%, 99 \%$ en la zona urbana y $72 \%$ en la rural; y para las instalaciones de saneamiento básico de $77 \%$, 82\% en la zona urbana y $63 \%$ en la rural (OPS, 2012).

La Superintendencia de Servicios Públicos Domiciliarios tiene registrados 203 prestadores del servicio de agua potable en Colombia, 125 suministraron agua apta para el consumo humano en el año 2011, con la calificación de Sin Riesgo Sanitario, lo que equivale a decir que el $75 \%$ de la población colombiana consume agua de este tipo (Sánchez, 2013).

De otro lado, según información del Departamento Nacional de Planeación (DNP, 2012), el Departamento del Meta cuenta con una población de 906.805 habitantes (681.708 habitantes en la cabecera y 225.097 habitantes en el resto), distribuidos en 29 municipios. El DNO también muestra cifras importantes relacionadas con el sector de agua potable y saneamiento básico: una cobertura de acueducto del $78.77 \%, 88.3 \%$ a nivel urbano y $37.5 \%$ a nivel rural; y de alcantarillado del $79.11 \%$, 93.3\% a nivel urbano y $17.7 \%$ a nivel rural (Marín, 2011); y una tasa de enfermedades trasmitidas por vectores, entre las cuales se puede mencionar 43.448 (dengue clásico), 110.480 (malaria Falciparum). Dichas enfermedades tienen relación con el sector del agua potable y el saneamiento básico del departamento (OPS, 2009).
Lo anterior sugiere que el crecimiento demográfico, sin el debido control técnico administrativo de sostenimiento y protección, hace difícil la consecución de las metas. Adicional, existe una diferencia abismal entre las coberturas y calidades del agua entre las zonas rurales y urbanas, su análisis comparativo muestra un aumento de la brecha para corregir, cubrir, equiparar y dar cumplimiento, dado que el departamento tiene una tendencia hacia lo rural. Por lo anterior, el objetivo del presente estudio fue conocer las perspectivas y la prospectiva en las coberturas de acueducto y alcantarillado en los municipios del Departamento del Meta, además de la calidad del agua y el tratamiento de las aguas residuales.

\section{Materiales y métodos}

\section{- Tipo de estudio y población}

Se realizó un estudio descriptivo - prospectivo a partir de la información proveniente de la Corporación para el desarrollo sostenible del área de manejo especial la Macarena (Cormacarena), de los 29 municipios del Departamento del Meta (Cormacarena, 2009). La información fue seleccionada y clasificada según la categoría y la cantidad de habitantes (MAVDT, 2002), en función de los datos porcentuales de las coberturas de acueducto y alcantarillados, también del Índice de Riesgo de la Calidad del Agua para consumo Humano (IRCA) y de la instalación de las Plantas de Tratamiento de Aguas Residuales (PTAR) (MAVDT, 2005).

\section{- Análisis de la información}

Se realizó un analisis estocástico, en términos de los datos de coberturas, IRCAs, y la indicación de la afirmación y negación de las PTAR. La presentación de los datos se realizó de forma cuantitativa y el análisis de los mismos, de forma cualitativa. 


\section{Resultados}

En la Figura 1 se muestra el comportamiento irregular de las coberturas de acueducto y alcantarillado (cabecera), para los 29 municipios en el Departamento del Meta incluida la capital. El promedio de la cobertura de acueducto para la cabecera municipal fue de $86.34 \%$, que presenta una diferencia del $2 \%$ con lo reportado por Marín (2011). Respecto a la continuidad del servicio de agua potable, el promedio Departamental es de 14.3 horas por día, según lo reportado por los municipios. Dicha continuidad tiene una diferencia de 7 horas por día con respecto al promedio nacional de 21.3 horas por día (CEPIS, 2000). El 46.4\% de los municipios reportaron una continuidad del servicio por encima del promedio antes mencionado; de estos, solo diez (10) municipios declararon continuidad del servicio de 24 horas por día, como debe ser la operación del servicio. El 53.6\% de los municipios reportaron continuidad del servicio por debajo del promedio antes señalado.

Con respecto al alcantarillado, la cobertura promedio en la cabecera fue de $85.5 \%$ que presenta una diferencia de 7.8\% con lo reportado por Marín (2011), diferencia considerable, ya que como infraestructura, el error establecido puede ser el año y el periodo del reporte de los datos y la conceptualización misma de cobertura. Figura 1.

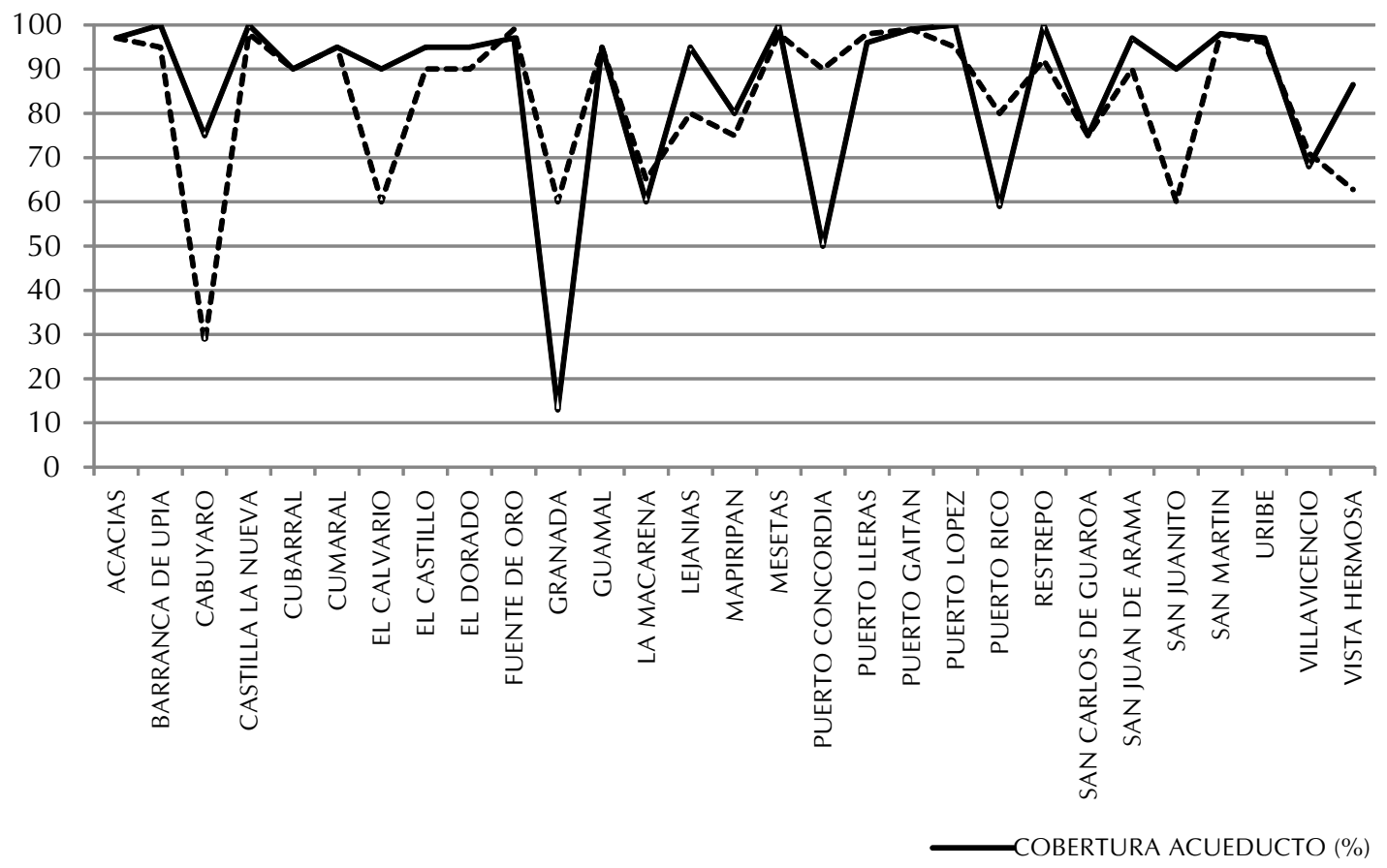

Figura 1. Coberturas de acueducto y alcantarillado, municipios del departamento.

En la Figura 2, se muestra la calidad del agua potable de acuerdo al índice de riesgo de la calidad del agua para consumo humano (IRCA), según el artículo 13 de la resolución 2115 de 2007. Se observa que el $43 \%$ (riesgo inviable del $4 \%$ y riesgo alto del $39 \%$ ) de los municipios, presentan una calidad del agua con nivel de riesgo Inviable - Alto. Solo el 39\% (riesgo bajo del $21 \%$ y $\sin$ riesgo del $18 \%$ ) de los municipios distribuyen agua con un nivel de riesgo bajo o sin riesgo para el consumo humano. 


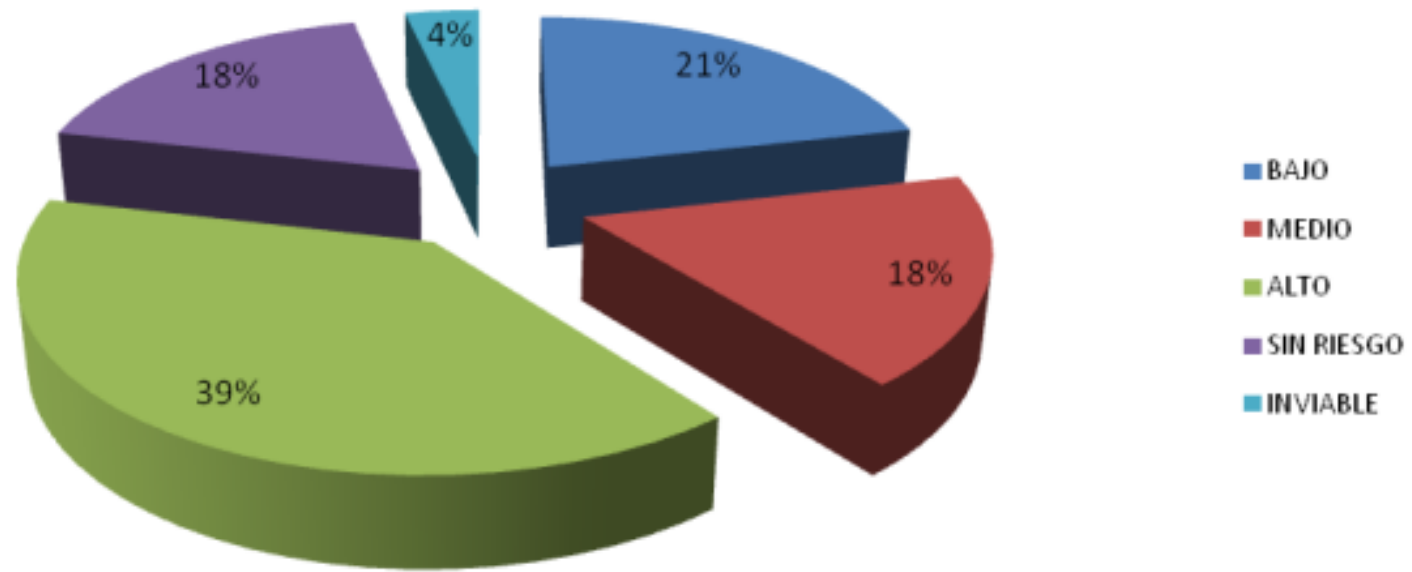

Figura 2. Calidad del agua en los municipios del departamento según el IRCA.

En la Figura 3, se muestran los municipios del departamento que tienen o no Planta de Tratamiento de Aguas Residuales (PTAR). Se observa que el 68\% de los municipios analizados no cuentan con una PTAR y solo el $32 \%$ de los municipios realizan tratamiento de aguas residuales.

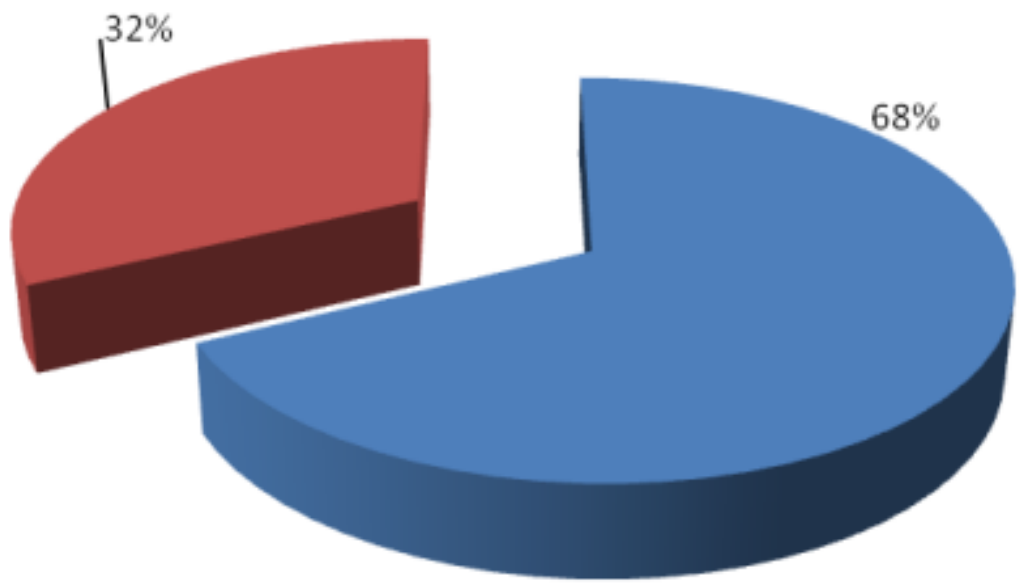

- NOTIENE PTAR

SI TIENE PTAR

Figura 3. Municipios en el departamento que tienen o no PTAR.

\section{Discusión}

En el sector de agua potable y saneamiento básico, se ha demostrado que existen problemas considerables de bajas coberturas de acueducto y alcantarillado, en algunas poblaciones del departamento (población menos de 100.000 habitantes), y también en municipios (cabecera entre 10.000 y 40.000 habitantes). Allí se presentan problemas en la calidad de los servicios públicos prestados, baja continuidad, mala calidad del agua, descarga de aguas residuales a cuerpos de agua sin tratamiento. También se presenta disposición de residuos sólidos en cuerpos de agua, bajo nivel en la calificación del recurso humano y un escaso desarrollo científico y tecnológico; lo que confirma una mala gestión de las entidades prestadoras de los servicios (Mc Guinness, 1997). 
La correlación de los resultados con elementos teóricos permite sugerir que el saneamiento básico ambiental en el departamento, como en la mayoría de regiones en los países en vía de desarrollo, presenta efectos sobre la salud pública como enfermedades de carácter hídrico en la población adyacente. Según Datos de la sección de Agua, Saneamiento e Higiene de la UNICEF (2009), 4200 niños fallecen por día debido a las enfermedades de carácter hídrico. Lo anterior se debe a que las soluciones técnicas son poco adecuadas o presentan sistemas de saneamiento poco eficientes para la recolección de las aguas residuales. Eso conlleva a los problemas de calidad en el servicio de las empresas prestadoras del servicio, y desconoce que las mejoras en el saneamiento generan una reducción de la morbilidad por enfermedades de transmisión fecal oral (Norman, 2010).

El $75 \%$ de los municipios del departamento reportó coberturas de acueducto (cabecera) superiores al promedio (86.34\%), y dentro de ellos, de manera especial, cinco municipios reportan coberturas del $100 \%$ en la cabecera. Por lo anterior, sería interesante corroborar la información; si verdaderamente los usuarios del servicio están conectados al acueducto. El $25 \%$ de los municipios reportan coberturas inferiores al promedio (86.34\%), lo cual es un indicador de un rezago en las inversiones, y a su vez, un indicador de no suministro del servicio de agua potable a la totalidad de la población, por lo menos en la cantidad necesaria (Rodríguez, 2009).

En materia de cobertura de alcantarillado, el $64.3 \%$ de los municipios reportan coberturas de alcantarillado superiores al promedio $(85.5 \%)$. El $35.7 \%$ reportan coberturas inferiores al promedio (85.5\%), lo cual es reflejo de una mala gestión de la autoridad municipal, o por lo menos una poca planeación. En cuanto a la calidad del agua potable en los municipios analizados, se observa que el $43 \%$ (riesgo inviable del $4 \%$ y riesgo alto del 39\%) de los municipios presentan una calidad del agua con nivel de riesgo Inviable - Alto. Significa que la calidad del agua distribuida por las empresas de servicios públicos no es apta para el consumo humano; es decir, es inviable sanitariamente y debe haber una gestión directa del prestador del servicio para realizar mejoras a la calidad del agua. El $18 \%$ presenta un riesgo medio, lo que indica que aún sigue siendo un agua no apta para consumo humano, y de igual forma tiene restricciones porque algunos parámetros no cumplen con la normatividad de agua potable.

Solo el 39\% de los municipios (riesgo bajo del $21 \%$ y sin riesgo del $18 \%$ ), distribuyen agua con un nivel de riesgo bajo o sin riesgo para el consumo humano, es decir, el agua potable se puede consumir sin problemas para la salud y desde luego se debe continuar con el seguimiento y monitoreo de la calidad del agua. Según lo anterior, en comparación con el resultado nacional (IRCA, nivel de riesgo medio, consolidado), se contaría con un agua potable de mejor calidad para consumo humano, solo en el 39\% de los municipios del departamento; sin embargo, para el resto de los municipios (61\%), se estaría muy por encima del consolidado nacional. En materia del tratamiento de aguas residuales domésticas mediante PTAR, se observa que el $68 \%$ de los municipios analizados no poseen una planta de tratamiento de sus aguas residuales, descargándola sin ningún control a los cuerpos de agua, que en muchos casos aguas abajo, toman el agua para el tratamiento del agua potable (Rodríguez, 2009). Eso está relacionado con el informe técnico sobre sistemas de tratamiento de aguas residuales en Colombia - línea base 2010 (Marín, 2012). Allí se establece que existe ausencia de seguimiento y control a los procesos; no se realizan caracterizaciones del agua, no se lleva un control del caudal de entrada y de salida, ni se conocen los caudales de diseño, de tal manera que la operación de los sistemas se efectúa de manera empírica, autónoma y rutinaria; falta de conocimiento de los sistemas 
operados, lo cual no permite planear con claridad la expansión y optimización de los sistemas de tratamiento; falta de mantenimiento correctivo y preventivo de los sistemas de tratamiento de aguas residuales; ausencia de programas de control de vectores y manejo de lodos; control sobre vertimientos de aguas residuales no residenciales y hacer seguimiento al cumplimiento de la normatividad.

Otro aspecto por destacar es que muchos de estos municipios realizan un esfuerzo en la recolección de las aguas residuales generadas en los centros poblados, pero después se pierde el esfuerzo, dado que no existe un tratamiento de sus aguas residuales. De otro lado, solo el $32 \%$ de los municipios realizan tratamiento de aguas residuales. Sin embargo, la cifra anterior del Departamento del Meta, en comparación con los valores en Colombia $(43,8 \%)$, está por debajo del promedio nacional, al igual que se encuentra por debajo del promedio latinoamericano (54\%). Ahora bien, en este caso, no es únicamente tratarla, sino cumplir con los niveles exigidos de la normatividad colombiana, es decir, tener una eficiencia adecuada en función de la operación y mantenimiento de las PTAR.

\section{Conclusiones}

En el Departamento del Meta se observan rezagos nominales en las coberturas de acueducto y alcantarillado. Corresponden al $21.23 \%$ y $20.89 \%$ respectivamente, lo cual es equivalente a decir que la inversión proyectada sería de US\$16.5 millones y US\$ 20 millones de dólares respectivamente, para alcanzar la meta del $100 \%$ de cobertura tanto en acueducto como en alcantarillado, sin afectar marcos fiscales nacionales y departamentales a mediano plazo. Lo anterior señala un fortalecimiento institucional y desde luego una mayor capacidad técnica, lo cual generaría una mayor continuidad del servicio (por lo menos cercana al promedio nacional); una mejor calidad del agua potable, es decir, un IRCA sin riego sanitario para el consumo humano; disminución de las enfermedades de carácter hídrico, y además, un tratamiento de las aguas residuales en la totalidad de los municipios mediante PTRAs. Desde luego, se debe tener en cuenta la selección adecuada de la tecnología, basada en variables como el clima, población, grado de complejidad, participación local, impactos ambientales, confiabilidad, flexibilidad y el costo de inversión, operación y mantenimiento, entre otros aspectos.

\section{Agradecimientos}

Los autores expresan los más sinceros agradecimientos a la Coordinación del programa de maestría en gestión ambiental sostenible de la Universidad de Los Llanos, así como al profesor de planta de la Universidad Distrital Francisco José de Caldas, Vidal Fernando Peñaranda Galvis por sus aportes y revisión de este documento.

\section{Referencias}

Bryman A. 2003. Social research methods. Second edition. New york: Oxford university press.

CEPIS. 2000. Evaluación de los servicios de agua potable y saneamiento en la región de las Américas. Lima: Centro Panamericano de Ingeniería Sanitaria y Ambiental.

CORMACARENA. 2009. Propuesta Plan Departamental de Aguas del Departamento del Meta. Documento técnico. Plan de gestión ambiental regional de CORMACARENA. Villavicencio: CORMACARENA.

Fry LM. Water, Human Health, and Sustainable Development. Water Quality and Purification. 2012; 4:299 - 314 
DNP. 2012. Regionalización del presupuesto de inversión. Departamento del Meta. Bogotá: Departamento Nacional de Planeación.

Marín C. Análisis del costo per capita de ampliacion de cobertura a nivel nacional de los servicios de acueducto y alcantarillado. Revista regulación de agua potable y saneamiento básico, 2011;153-190.

MAVDT. 2002. Gestión integral del agua. Bogotá: Ministerio de ambiente, vivienda y desarrollo territorial.

MAVDT. 2005. Sistema de gestión de calidad. Ventanilla única. Bogotá: Ministerio de ambiente, vivienda y desarrollo territorial.

Mc Guinness T. The diversification strategies of the privatised water and sewerage companies in england and water: a resource based view. Utilities policy, 1997; $325-339$.

Mugabi JE. Strategic planning for water utilities in devoloping countries. Utilities policy, $2007 ; 1-8$.

Nogueira M. Performance measurement and indicators for water supply management: Review and international cases. Renewable and Sustainable Energy Reviews. 2015; 1-12.

Norman G. Effects of sewerage on diarrhoea and enteric infections: a systematic review and meta-analysis. Lancet Infect. 2010;536 $-544$.

Noyola A. En latinoamérica tratamiento de aguas residuales domésticas. Revista de ingeniería sanitaria y ambiental ACODAL, 2004; 20 - 25.

OPS. (2009). Indicadores básicos. Situación de salud de las Américas. Bogotá:
Organización panamerica de la salud.

OPS. 2012. Bogotá: Organización Panamericana de la Salud.

Roelich K. Towards resource-efficient and service-oriented integrated infrastructure operation. Technological Forecasting \& Social Change. 2015; 40-52.

Rodríguez JP. Utilización del agua de la red de abastecimiento público para embotellarla. Tecnología del agua. 2009; 64 - 65.

Rodríguez JP. Selección técnico económica del sistema de depuración de aguas, aplicado a la evaluación de la descontaminación hídrica. Tecnología del agua. 2009; 22 - 31 .

Rodríguez JP. 2010. Contaminación del agua. En Contaminación ambiental en Colombia. (págs. 255 - 300). Bogotá: Fundación en causa por el desarrollo humano.

Sánchez J. 2013. Estado del sector de acueducto y alcantarillado. Estado del sector de acueducto y alcantarillado. Santa Marta: Superintendencia de servicios públicos domiciliarios. Pp1-30. 